Research Article

\title{
Load Identification Method Based on Interval Analysis and Tikhonov Regularization and Its Application
}

\author{
Chunsheng Liu, ${ }^{1}$ Chunping Ren $\mathbb{D}^{1,2}$ and Nengjian Wang ${ }^{2}$ \\ ${ }^{1}$ Heilongjiang University of Science and Technology, Harbin 150022, China \\ ${ }^{2}$ College of Mechanical and Electrical Engineering, Harbin Engineering University, Harbin 150001, China \\ Correspondence should be addressed to Chunping Ren; renchunpin@sina.com
}

Received 15 February 2019; Revised 30 April 2019; Accepted 8 July 2019; Published 17 July 2019

Academic Editor: Ephraim Suhir

Copyright $($ C 2019 Chunsheng Liu et al. This is an open access article distributed under the Creative Commons Attribution License, which permits unrestricted use, distribution, and reproduction in any medium, provided the original work is properly cited.

In order to study the dynamic force identification method of an end-plate pick of shearer, a dynamic force identification technique based on interval theory was proposed. The dynamic force interval identification model is established by describing and quantifying the identified parameters. By using the interval analysis method of the first-order Taylor expansion, the dynamic force identification is transformed into two kinds of deterministic inverse problems at the midpoint of the uncertain parameter and its gradient identification. The Tikhonov regularization method is used to solve two kinds of deterministic problems, and the upper and lower boundaries of dynamic force of the end-plate pick are determined. The results show that the deviations between the identified dynamic force and the actual dynamic force are basically within $2 \%$ and $5 \%$, and the average uncertainties are up to $7 \%$ and $10 \%$. Therefore, the proposed method can effectively determine the upper and lower boundaries of dynamic force of the endplate pick, improve the solving efficiency, and provide a new research method for studying the coal rock mechanism of the pick cutting load.

\section{Introduction}

In addition to the structural parameters and motion parameters of the pick, the load of coal and rock is also affected by uncertain parameters such as coal quality parameters and geological conditions. As a result, the established forward load identification model and the actual load inevitably have certain approximation and uncertainty, which makes the uniqueness, existence, and stability of the load identification solution impossible to adopt certainty [1-3]. Therefore, it is of great theoretical value and practical significance to correctly and objectively estimate the impact of uncertainty on load identification results.

At present, there are mainly probability methods, such as fuzzy method and interval method, for describing uncertain information. Among them, it is most common to transform uncertain parameters into mathematical expressions of random variables and analyze uncertainties based on probability and statistics theory. However, for most practical engineering problems, due to the limitations of measurement technology, economy, and other practical conditions, it is often difficult to obtain enough samples, which makes it difficult for this method to obtain satisfactory identification load. For the method of fuzzy theory, in practical problems, the degree of fuzzy membership is determined by the experience of limited samples and decision-makers, which has a lot of subjective factors, leading to the use of the fuzzy method to identify whether loads will bring greater errors. For practical engineering problems, it is usually much easier to obtain the possible bounds of system uncertainties than its statistics. In the study of Liu et al. [4], an efficient interpolation-based method is proposed to reduce ill-posedness availably and identify dynamic load stably. Numerical examples demonstrate the effectiveness of the proposed method and the correctness of identified load. In the study of Li et al. [5], a laboratory method based on equivalent dynamic load reconstruction is proposed for the identification of oil-film coefficients, a rotor structure with two journal bearings is investigated, and the identification results of oil-film coefficients demonstrate the validity and accuracy of the 
proposed method. The interval theory method only needs to know the bounds of uncertain parameters, and the result is a minimum set of all feasible solutions [6-8]. So far, this uncertainty description method is more suitable for solving engineering problems.

Domestic and foreign scholars have made some achievements in the research of load identification based on the interval analysis method. Wang and Matthies [9] proposed a novel model calibration method that combines nonprobabilistic interval technology with Bayesian analysis theory, and the feasibility of the proposed method is demonstrated using the renowned Sandia thermal challenge problem. Wang and Matthies and Wang et al. [10, 11] proposed a novel model validation approach by using evidence theory. In the study of Ahmari and Yang [12], interval variables are used to represent rock mechanics parameters, and an interval back analysis model for identifying rock mechanics parameters is established. In the study of Jiang et al. [13], an interval inversion model is established to predict the spring coefficient and mass range of the system. In the study of Lee et al. [14], based on the interval analysis method, an interval inversion model for modal parameter modification is established. In the study of Li et al. [15], the identification of material parameters of composite laminates with uncertain parameters is studied based on interval analysis method. In the study of $\mathrm{Au}$ et al. [16], a method combining interval analysis with regularization is proposed to identify the dynamic loads acting on uncertain structures, and the interval boundaries of the loads to be identified are obtained by interval expansion. Han et al. [17] presented an interval method for dynamic load identification based on Green's function and obtained the interval estimation bounds of the identified dynamic loads in time domain. Averbakh and Lebedev [18] discussed the effects of heterogeneity, interval radius of uncertain parameters, initial value selection, and data noise on inversion results. In the study of Rodriguez et al. [19], homotopy technique is introduced into load identification problem, and based on the interval finite element method and matrix perturbation theory, the inverse problem of transient heat conduction is studied. In the study of Link et al. [20], based on the interval finite element method and adjoint optimization, an interval method for load identification of structural static parameters is proposed. In the study of Liu et al. [21], based on the Bayesian method and interval analysis method, a hybrid inversion method for parameter identification in uncertain environment is proposed. In the study of Song et al. [22], a load identification method based on the shape function method and interval analysis method is proposed.

To sum up, the research of the interval theory analysis method is not perfect in practical engineering application. The main problems at this stage are described as follows: the quantification and propagation methods of uncertainties based on poor information are still unclear, and the inversion effect of the interval inversion algorithm is different for different research objects, and there is no unified theoretical model. In the field of mining machinery, its interval theory method is still in the stage of exploration and research, especially the research on load identification of end-plate pick is rarely reported at home and abroad. Because of the bad arrangement of end-plate pick and cutting environment, the current research on end-plate pick load is not mature and perfect [23-27]. Therefore, on the basis of previous research on blade load identification, it has certain reference value for the research of drum load identification and deduction.

\section{Interval Model of Dynamic Force Identification Problem}

When some parameters of the structure are uncertain, the convolution integral of the dynamic response of the structure can be written as follows [28]:

$$
y(t)=\int_{0}^{t} p(\tau, \lambda) g(t-\tau, \lambda) d \tau,
$$

where $\lambda$ is a vector describing structural uncertainty and the kernel function $g(t, \lambda)$ is a set compatible with the uncertainty parameter $\lambda$.

Since the solution of formula (1) is not a single solution, but a set of solutions, it is difficult for conventional deterministic methods to solve such problems directly. Therefore, based on the theory of interval mathematics, a new attempt is made to identify the dynamic force.

Interval numbers are defined as a pair of ordered real numbers [29]:

$$
A^{I}=\left[A^{L}, A^{R}\right]=\left\{A \mid A^{L} \leq A \leq A^{R}, A \in R\right\},
$$

where $I, L$, and $R$ are expressed as interval, lower bound, and upper bound, respectively.

For load identification of uncertain structures, the uncertain vector $\lambda^{I}$ in formula (1) can be modeled by interval vector $\lambda$ :

$$
\begin{aligned}
\lambda \in \lambda^{I} & =\left[\lambda^{I}, \lambda^{R}\right], \\
\lambda_{j} \in \lambda_{j}^{I} & =\left[\lambda_{j}^{I}, \lambda_{j}^{R}\right], \quad j=1,2, \ldots, n .
\end{aligned}
$$

For the same measurement response, since all possible values of structural uncertainties belong to intervals $\lambda^{I}$, the dynamic force identified at each moment $t$ is not a specific value but a bounded interval corresponding to all possible structural uncertainties:

$$
p(t, \lambda) \in p^{I}(t)=\left[p^{L}(t), p^{L}(t)\right]
$$

where $p^{I}(t), p^{L}(t)$, and $p^{R}(t)$ are the interval of dynamic force, the lower bound, and the upper bound of the interval.

According to the theory of interval mathematics, formula (3) can be expressed as follows:

$$
\begin{aligned}
\lambda^{I} & =\left[\lambda^{I}, \lambda^{R}\right] \\
& =\left[\lambda^{c}-\lambda^{w}, \lambda^{c}+\lambda^{w}\right] \\
& =\lambda^{c}+[-1,1] \lambda^{w}
\end{aligned}
$$

where $\lambda^{c}$ and $\lambda^{w}$ denote the midpoint and radius of the interval, respectively, which can be expressed as follows: 


$$
\begin{aligned}
& \lambda^{c}=\frac{\lambda^{L}+\lambda^{R}}{2}, \\
& \lambda_{j}^{c}=\frac{\lambda_{j}^{L}+\lambda_{j}^{R}}{2}, \quad j=1,2, \ldots, n, \\
& \lambda^{w}=\frac{\lambda^{R}-\lambda^{L}}{2}, \\
& \lambda_{j}^{w}=\frac{\lambda_{j}^{R}-\lambda_{j}^{L}}{2}, \quad j=1,2, \ldots, n .
\end{aligned}
$$

The level of uncertainty in the interval $\lambda^{I}$ is defined as follows:

$$
\gamma\left(\lambda^{I}\right)=\frac{\lambda^{w}}{\left|\lambda^{c}\right|} \times 100 \%
$$

According to formulas (1) and (5), the uncertainty vector $\lambda$ can be described as follows:

$$
\lambda=\lambda^{c}+\delta \lambda
$$

where $\delta \lambda \in[-1,1] \lambda^{w}$ and $\delta \lambda_{j} \in[-1,1] \lambda_{j}^{w}$.

Assuming that the uncertainties of all variables in $\lambda$ are small, the first-order Taylor expansion for identifying the dynamic force $p(t, \lambda)$ at the midpoint $\lambda^{w}$ of the interval is obtained:

$$
\begin{aligned}
p(t, \lambda) & =p\left(t, \lambda^{c}+\delta \lambda\right) \\
& \approx p\left(t, \lambda^{c}\right)+\sum_{j=1}^{n}\left|\frac{\partial p\left(t, \lambda^{c}\right)}{\partial \lambda_{j}}\right| \delta \lambda_{j},
\end{aligned}
$$

where $p\left(t, \lambda^{c}\right)$ denotes the identification of dynamic force when the uncertainty parameter is the interval midpoint value.

$\left(\partial p\left(t, \lambda^{c}\right)\right) / \partial \lambda_{j}$ denotes the first derivative of dynamic force to parameter $\lambda_{j}$, and the sensitivity of random load to parameter $\lambda_{j}$. Combinations (8) and (9) are obtained.

$$
\begin{aligned}
p(t, \lambda) & =p\left(t, \lambda^{c}+\delta \lambda\right) \\
& \approx p\left(t, \lambda^{c}\right)+\sum_{j=1}^{n}\left|\frac{\partial p\left(t, \lambda^{c}\right)}{\partial \lambda_{j}}\right|[-1,1] \lambda_{j}^{w} .
\end{aligned}
$$

To extend the restricted applicability of the traditional perturbation method with small uncertainty level, reference [30] presents a first-order subinterval parameter perturbation method (FSPPM) and a modified subinterval parameter perturbation method (MSPPM) to solve the problem with large interval parameters in material properties, external loads, and boundary conditions. Based on the subinterval theory, the original uncertain-but-bounded parameters with limited information are divided into several small subintervals. The eventual response interval is assembled by the interval union operation. In both methods, the Taylor series is used to approximate the interval matrix and vector.

Therefore, the interval model of dynamic force identification, and the upper and lower bounds of dynamic force identification, is obtained from equation (10):

$$
\begin{aligned}
p^{L}(t) & =\min _{\lambda \in \lambda^{I}} p(t, \lambda) \\
& =p\left(t, \lambda^{c}\right)-\sum_{j=1}^{n}\left|\frac{\partial p\left(t, \lambda^{c}\right)}{\partial \lambda_{j}}\right| \lambda_{j}^{w}, \\
p^{R}(t) & =\max _{\lambda \in \lambda^{I}} p(t, \lambda) \\
& =p\left(t, \lambda^{c}\right)+\sum_{j=1}^{n}\left|\frac{\partial p\left(t, \lambda^{c}\right)}{\partial \lambda_{j}}\right| \lambda_{j}^{w} .
\end{aligned}
$$

From formulas (11) and (12), it can be seen that the upper and lower bounds of identifying dynamic force for uncertain structures are transformed into two kinds of identifying deterministic dynamic force. The first is to identify the dynamic force $p\left(t, \lambda^{c}\right)$ at the midpoint of the uncertainty parameter. The other is to identify the first-order partial derivative $\left(\partial p\left(t, \lambda^{c}\right)\right) / \partial \lambda_{j}$ of the dynamic force for each uncertain parameter, that is, the sensitivity curve of each uncertain parameter.

\section{Method for Solving Interval Model}

Considering the influence of noise, formula (1) can be expressed in the following form:

$$
Y_{\mathrm{err}}=G P_{\text {true }}+\mathrm{err} \text {, }
$$

where $Y_{\mathrm{err}}$ is the measurement response with noise, $G$ is the kernel function, err is the unknown noise, and $e=l$ std $(y(t))$ rand, where $l$ denotes the parameter level, std (.) denotes the standard deviation, and rand denotes a random number which ranges from -1 to 1 .

Singular value decomposition (SVD) of the kernel function matrix is carried out, and the result is obtained:

$$
G=\mathbf{U} \sum \mathbf{V}^{T}=\sum_{i=1}^{n} \sigma_{i} u_{i} v_{i}^{T},
$$

where $\mathbf{U}=\left(u_{1}, u_{2}, \ldots, u_{n}\right)$ and $\mathbf{V}=\left(v_{1}, v_{2}, \ldots, v_{n}\right)$ are the column orthogonal matrices consisting of left and right singular vectors, respectively. And $\sum$ is a diagonal matrix constructed by the singular value of matrix $G . \sum=\operatorname{diag}\left(\sigma_{1}, \sigma_{2}, \ldots, \sigma_{n}\right)$ and $\sigma_{1} \geq \sigma_{2} \geq \cdots \geq \sigma_{n} \geq 0$.

Therefore, the identified dynamic force can be expressed in the following form:

$$
\begin{aligned}
\widetilde{P}=G^{-1} Y_{\text {err }} & =V \operatorname{Diag}\left(\sigma_{i}^{-1}\right) U^{T} Y_{\text {err }} \\
& =P_{\text {true }}+\sum_{i=1}^{n} \sigma_{i}^{-1}\left(u_{i}^{T} \text { err }\right) v_{i}
\end{aligned}
$$

where $\widetilde{P}$ is the identified load.

By using the Tikhonov method, the following formulas can be obtained:

$$
\left(G^{\mathrm{T}} G+\alpha^{2} I\right) P=G^{\mathrm{T}} Y_{\mathrm{err}}
$$

where $\alpha$ is a regular parameter, which can be obtained by the GCV method. 
Combining formulas (14) and (15), we get

$$
P=\sum_{i=1}^{n} \frac{\sigma_{i}^{2}}{\sigma_{i}^{2}+\alpha^{2}} \frac{u_{i}^{T} Y_{\mathrm{err}}}{\sigma_{i}} v^{i} .
$$

Therefore, the dynamic force $p\left(t, \lambda^{c}\right)$ at the midpoint of the uncertain parameter can be obtained by using the above method. The finite difference method is used to calculate the first-order partial derivatives of uncertain parameters. After identifying the two deterministic problems, the upper and lower bounds of the final dynamic force can be identified by using formulas (11) and (12).

\section{Result Analysis}

The interval theory method is applied in the identification of dynamic force of end-plate pick, and the upper and lower bounds of identification dynamic force are obtained, so as to provide theoretical reference and method for the study of drum load deduction.

4.1. Testing System. The test system for dynamic force of coal and rock cut by pick is shown in Figure 1. The cutting motor drives the cutting arm to rotate through the reducer and the rotating speed torque meter. The speed of the cutting arm is regulated by the frequency conversion method. The feed motion of the cutting test bed is realized by the hydraulic cylinder. Feedback from the speed sensor can automatically and manually adjust the speed.

The load testing system of the pick is composed of the force measuring device, pressure sensor, signal amplifier, Dasp V10 intelligent data acquisition, and signal processing system. The parameters are as follows: the rated power of the frequency conversion motor is $55 \mathrm{~kW}$, the cutting device can simulate the speed range of the shearer drum which is 0 $48 \mathrm{r} / \mathrm{min}$, the force sensor is $0-5000 \mathrm{~N}$, the measuring range of the torque is $0-22000 \mathrm{~N} \cdot \mathrm{m}$, the cutting diameter is $1200-2000 \mathrm{~mm}$, and the traction speed is $0.5-2 \mathrm{~m} / \mathrm{min}$. In the process of rotary cutting, the dynamic force on the pick is converted into electrical signals through the deformation of five pressure sensors, and the signals are transmitted to Dasp V10 intelligent data acquisition and signal processing system through multiple sliding rings.

The experimental conditions are as follows: the arrangement of the end-plate pick is chessboard, the installation angle of pick is $40^{\circ}$, the axial inclination angle is $10^{\circ}$, the secondary rotation angle is $0^{\circ}$, the maximum cutting thickness is $20 \mathrm{~mm}$, the speed of the cutting arm is $40.8 \mathrm{r} /$ $\mathrm{min}$, and the traction speed is $0.82 \mathrm{~m} / \mathrm{min}$. The measured axial load of the pick is shown in Figure 2. The measurement response is shown in Figure 3. The kernel function is shown in Figure 4.

4.2. Identified Result. Because of the complex genesis of coal and rock, uneven medium, and anisotropy, the density of coal and rock is uncertain. Therefore, the density of coal and rock is regarded as an uncertain parameter, in which the point value is $1 \mathrm{~g} / \mathrm{cm}^{3}$, and the intervals of four uncertainty

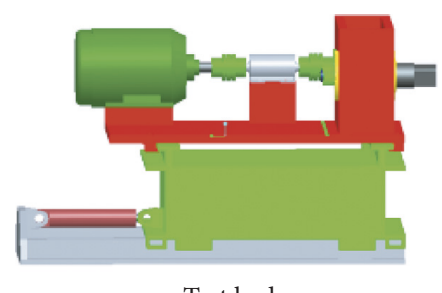

Test bed

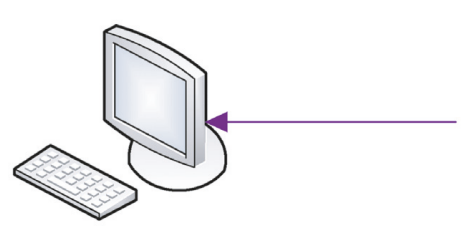

Signal acquisition system

Figure 1: Test system.

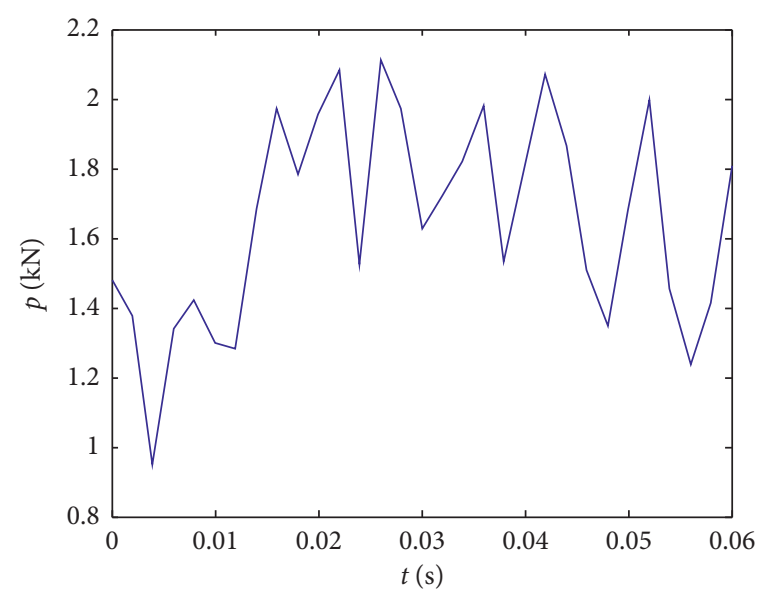

Figure 2: Cutting load.

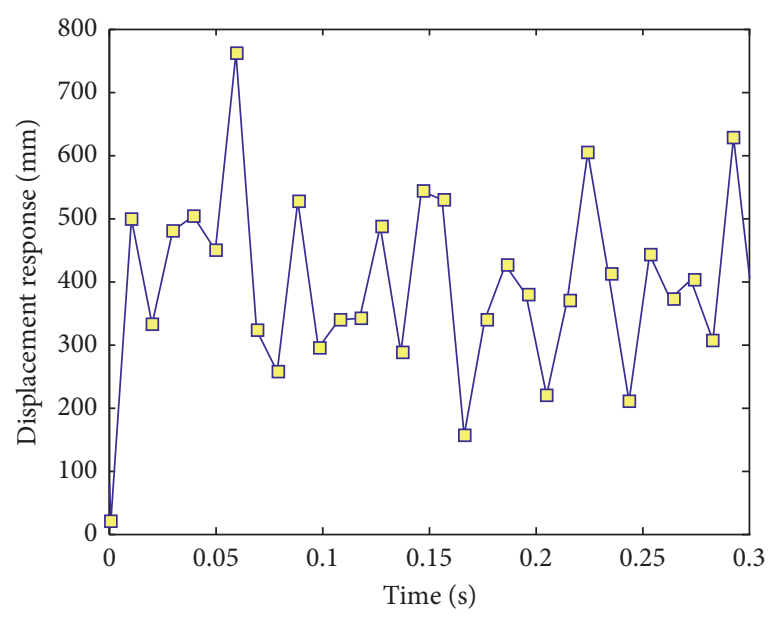

Figure 3: Measurement response.

levels, such as $2 \%, 5 \%, 7 \%$, and $10 \%$, are taken as shown in Table 1.

In addition, in order to quantitatively judge the effect and quality of dynamic force identification, the following evaluation indexes are defined $[31,32]$ : 


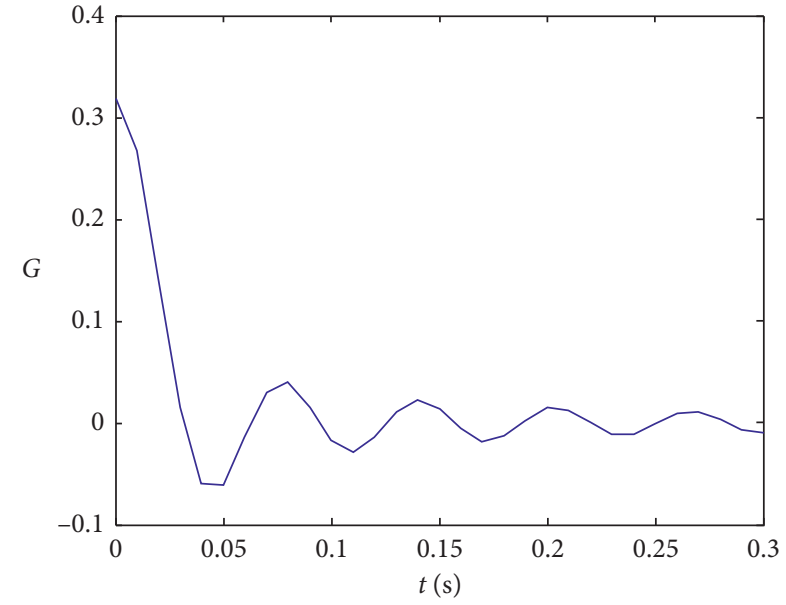

FIgURE 4: Kernel function.

TABLE 1: Interval range of uncertain parameters.

\begin{tabular}{lcccc}
\hline $\begin{array}{l}\text { Uncertainty } \\
\text { parameter }\end{array}$ & $2 \%$ & $5 \%$ & $7 \%$ & $10 \%$ \\
\hline Density $\left(\mathrm{g} / \mathrm{cm}^{3}\right)$ & {$[0.98,1.02]$} & {$[0.95,1.05]$} & {$[0.93,1.07]$} & {$[0.90,1.10]$} \\
\hline
\end{tabular}

$$
s=\frac{\left\|p^{L}-p^{R}\right\|}{\left\|p_{\text {true }}\right\|}
$$

where $s$ is the relative error.

Firstly, the cutting load is identified by taking the midpoint value of the uncertainty parameter and using the regularization method. As shown in Figure 5, the cutting load at the midpoint of the interval is identified.

Secondly, in order to obtain the sensitivity curve of the cutting random load to uncertain parameters (coal rock density), the central difference method is used to solve the problem. The step length is $5 \%$ of the midpoint value, and the sensitivity curve of parameters (coal rock density) is obtained, as shown in Figure 6. This curve shows that the uncertain parameters have an important influence on the cutting load. Firstly, the uncertain parameters determine the performance of the cutting structure and then determine the cutting parameters.

It can be seen from the figure that the cutting random load is relatively sensitive to the density of coal and rock. Loads are calculated by formulas (11) and (12) after obtaining the sensitivity curves of cutting loads at the midpoint and the uncertainty resistance parameters of coal and rock density. The uncertainty level determines the range of the area surrounded by the upper and lower bounds of the cutting load.

The higher the uncertainty level, the greater the radius of the interval. The uncertainty parameters can take a wider sample point, so the upper and lower bounds of the identified cutting load will surround a wider region. Therefore, the identification of upper and lower bounds of cutting loads is studied under four uncertainties. The upper and lower bounds of identified results are shown in Figures $7(a)-7(d)$.

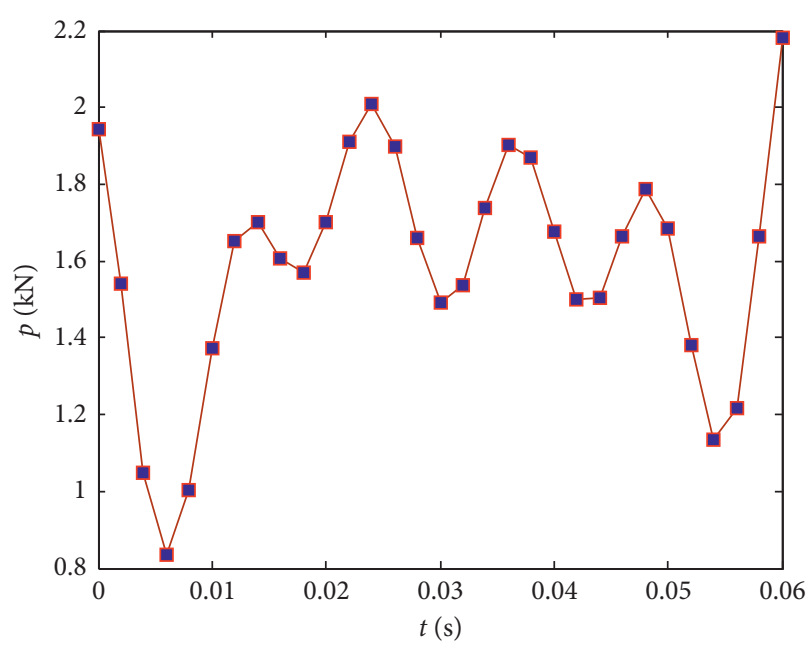

FIGURE 5: Identified result at the midpoint of interval.

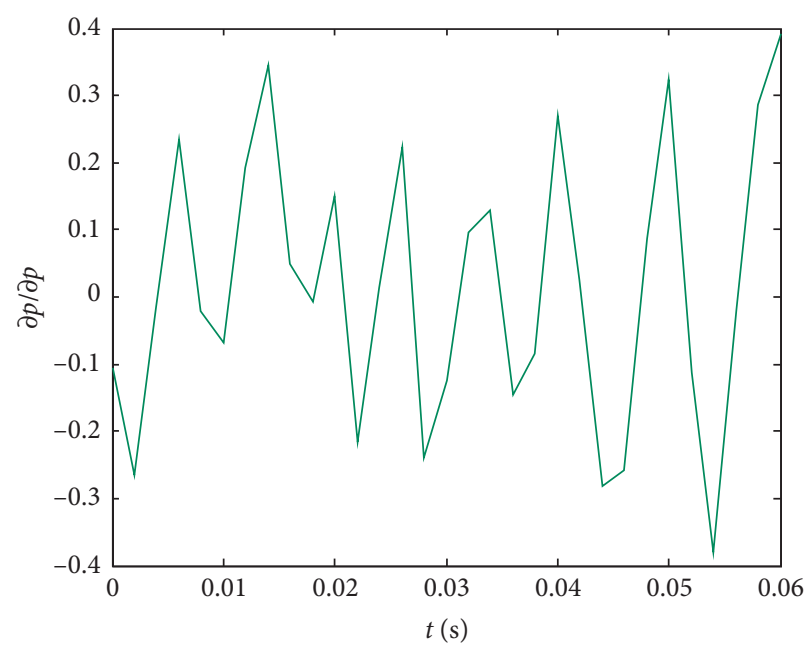

FIgURE 6: Sensitivity curve of the load on cutting impedance.

From the identification results of four uncertain horizontal cutting loads, the upper and lower bounds of identification loads are expanding with the increase of uncertainty level. Because the interval analysis method is limited to a small level of uncertainty, the upper and lower bounds of the identified cutting load are increasingly unsatisfactory with the increase of the level of uncertainty.

Figures 7(a) and 7(b) show that the identification results of the interval analysis method contain real cutting loads within $5 \%$ uncertainty level. It can be seen that the interval analysis method can ensure higher identification accuracy at a lower uncertainty level. The advantage of interval analysis is obvious in computational efficiency, which also shows the correctness and effectiveness of interval analysis at low uncertainty level.

Figures $7(\mathrm{c})$ and $7(\mathrm{~d})$ show that the upper and lower bounds of the load interval expand more and more with the increase of the uncertainty level above the 5\% uncertainty level. In order to further evaluate the effect of load identification at different uncertain levels, the evaluation index values are given, as shown in Table 2. 


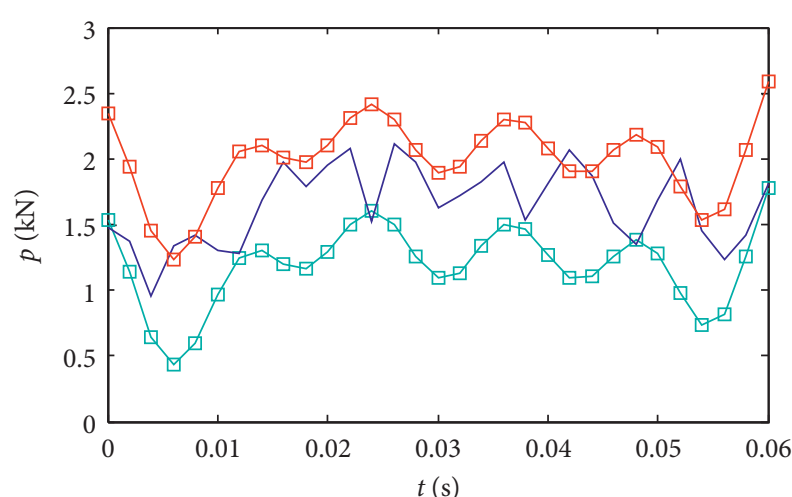

$\square-$ Lower boundary of identified dynamic force

- Actual dynamic force

$\square$ Upper boundary of identified dynamic force

(a)

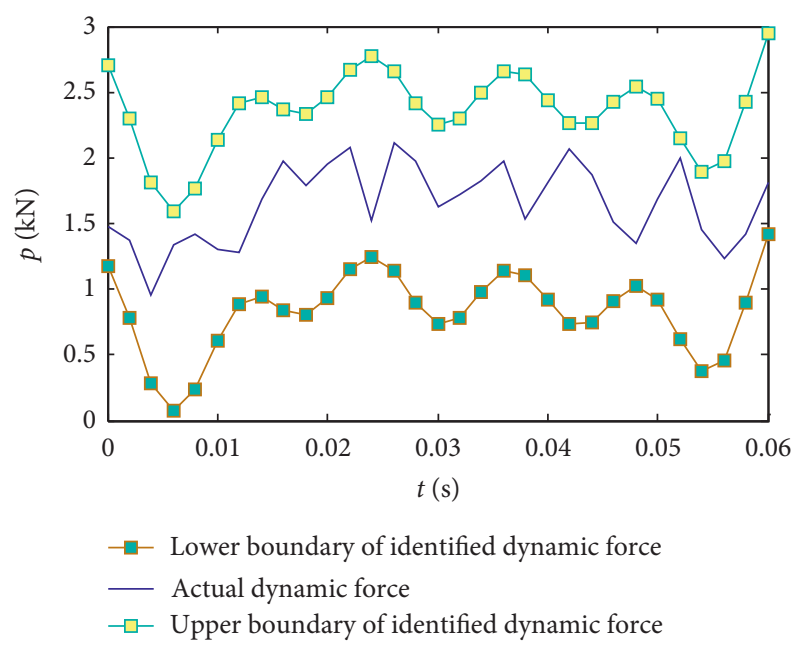

(c)

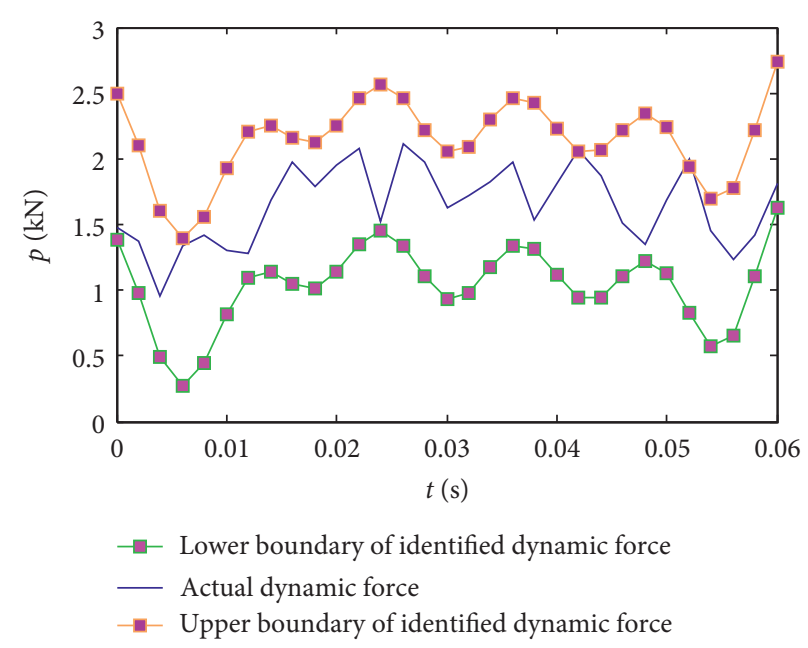

(b)

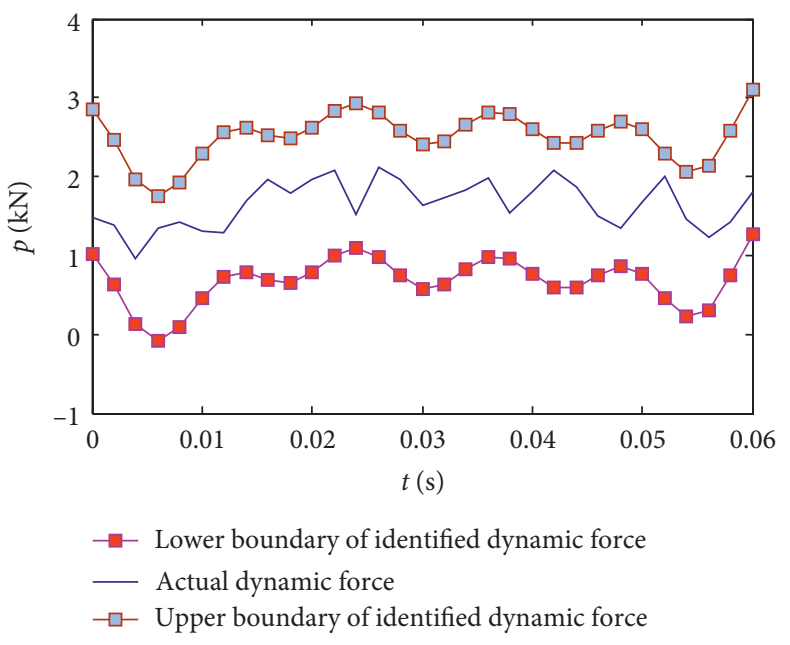

(d)

Figure 7: Identified results of different uncertainty levels: (a) 2\%, (b) 5\%, (c) 7\%, and (d) 10\%.

TABLE 2: The evaluation index.

\begin{tabular}{lcccc}
\hline Uncertainty level & $2 \%$ & $5 \%$ & $7 \%$ & $10 \%$ \\
\hline Interval analysis & 0.02 & 0.05 & 0.07 & 0.10 \\
\hline
\end{tabular}

From Table 2, it can be seen that when the uncertainty level is $2 \%$ and $5 \%$, the identification cutting load index values of the interval recognition algorithm are 0.02 and 0.05 , respectively. When the uncertainty level is more than $5 \%$, the index value increases with the increase of the uncertainty level. When the uncertainty level is $10 \%$, the difference between the index value and $2 \%$ is more than two times. This fully shows that the upper and lower bounds of the cutting load identified by the interval analysis method have deviated more seriously at this moment, and the identification results are not satisfactory and have no reference value.

In summary, the identification effect of the cutting load is better when the uncertainty level is $2 \%$. That is to say, the interval theory analysis method has better identification effect and higher identification efficiency at low uncertainty level. At the same time, it provides a theoretical reference for the study of drum load identification.

\section{Conclusion}

(1) An effective method for identifying dynamic force of the end-plate pick of shearer is proposed. The identified parameters are described and quantified by intervals. Using the interval analysis method of the first-order Taylor expansion, the dynamic force identification is transformed into two kinds of deterministic inverse problems of midpoint and gradient identification of uncertain parameters, and then the mathematical model of dynamic force interval identification is established.

(2) When the uncertainty level is $2 \%$ and $5 \%$, the identification dynamic force index values of the interval analysis identification algorithm are 0.02 and 0.05 , respectively. When the uncertainty level is more than $5 \%$, the index value increases with the increase of the uncertainty level. When the uncertainty level is 
$7 \%$ and $10 \%$, the difference between the index value and $2 \%$ is twice. Therefore, when the uncertainty level is $2 \%$, the effect of dynamic force identification is better.

\section{Data Availability}

No data were used to support this study.

\section{Conflicts of Interest}

The authors declare that they have no conflicts of interest.

\section{Acknowledgments}

This work was supported by the Chinese National Natural Science Foundation (contract nos. 51674106 and 51274091).

\section{References}

[1] S. S. Rao and L. Berke, "Analysis of uncertain structural systems using interval analysis," AIAA Journal, vol. 35, no. 4, pp. 727-735, 1997.

[2] Z. Jianguo, C. Jianjun, and M. Xiaosong, "Discussion on the method for interval analysis of uncertain structural systems by using universal grey numbers," Mechanical Science and Technology, vol. 24, no. 11, pp. 1272-1276, 2005.

[3] N. Impollonia and G. Muscolino, "Interval analysis of structures with uncertain-but-bounded axial stiffness," Computer Methods in Applied Mechanics and Engineering, vol. 200, no. 21-22, pp. 1945-1962, 2011.

[4] J. Liu, X. Meng, D. Zhang, C. Jiang, and X. Han, “An efficient method to reduce ill-posedness for structural dynamic load identification," Mechanical Systems and Signal Processing, vol. 95, pp. 273-285, 2017.

[5] K. Li, J. Liu, X. Han, C. Jiang, and H. Qin, "Identification of oil-film coefficients for a rotor-journal bearing system based on equivalent load reconstruction," Tribology International, vol. 104, pp. 285-293, 2016.

[6] G. Muscolino and A. Sofi, "Analysis of structures with random axial stiffness described by imprecise probability density functions," Computers \& Structures, vol. 184, pp. 1-13, 2017.

[7] H. Nguyen-Xuan, L. V. Tran, C. H. Thai, and T. Nguyen-Thoi, "Analysis of functionally graded plates by an efficient finite element method with node-based strain smoothing," ThinWalled Structures, vol. 54, pp. 1-18, 2012.

[8] L. V. Tran, C. H. Thai, and H. Nguyen-Xuan, "An isogeometric finite element formulation for thermal buckling analysis of functionally graded plates," Finite Elements in Analysis and Design, vol. 73, pp. 65-76, 2013.

[9] C. Wang and H. G. Matthies, "Novel interval theory-based parameter identification method for engineering heat transfer systems with epistemic uncertainty," International Journal for Numerical Methods in Engineering, vol. 115, no. 6, pp. 756770, 2018.

[10] C. Wang and H. G. Matthies, "Novel model calibration method via non-probabilistic interval characterization and Bayesian theory," Reliability Engineering \& System Safety, vol. 183, pp. 84-92, 2019.

[11] C. Wang, H. G. Matthies, M. Xu, and Y. Li, "Evidence-theorybased model validation method for heat transfer system with epistemic uncertainty," International Journal of Thermal Sciences, vol. 132, pp. 618-627, 2018.
[12] S. Ahmari and M. Yang, "Impact location and load identification through inverse analysis with bound uncertain measurements," Smart Materials \& Structures, vol. 22, no. 8, article 085024, 2013.

[13] C. Jiang, X. Han, and G. R. Liu, "Optimization of structures with uncertain constraints based on convex model and satisfaction degree of interval," Computer Methods in Applied Mechanics and Engineering, vol. 196, no. 49-52, pp. 47914800, 2007.

[14] H. W. Lee, G. A. Lee, D. J. Yoon, S. Choi, K. H. Na, and M. Y. Hwang, "Optimization of design parameters using a response surface method in a cold cross-wedge rolling," Journal of Materials Processing Technology, vol. 201, no. 1-3, pp. 112-117, 2008.

[15] G. Li, H. Wang, S. R. Aryasomayajula, and R. V. Grandhi, "Two-level optimization of airframe structures using response surface approximation," Structural and Multidisciplinary Optimization, vol. 20, no. 2, pp. 116-124, 2000.

[16] F. T. K. Au, Y. S. Cheng, L. G. Tham, and G. W. Zeng, "Robust design of structures using convex models," Computers \& Structures, vol. 81, no. 28-29, pp. 2611-2619, 2003.

[17] X. Han, D. Xu, and G. R. Liu, "Transient responses in a functionally graded cylindrical shell to a point load," Journal of Sound and Vibration, vol. 251, no. 5, pp. 783-805, 2002.

[18] I. Averbakh and V. Lebedev, "On the complexity of minmax regret linear programming," European Journal of Operational Research, vol. 160, no. 1, pp. 227-231, 2005.

[19] J. F. Rodriguez, J. E. Renaud, B. A. Wujek, and R. V. Tappeta, "Trust region model management in multidisciplinary design optimization," Journal of Computational and Applied Mathematics, vol. 124, pp. 139-154, 2000.

[20] T. L. Link, J.-S. Lew, and L. H. Keel, "Interval model identification and robustness analysis for uncertain flexible structures," IEEE Transactions on Control Systems Technology, vol. 4, no. 4, pp. 411-418, 1996.

[21] J. Liu, X. Sun, X. Meng, K. Li, G. Zeng, and X. Wang, “A novel shape function approach of dynamic load identification for the structures with intereval uncertainty," International Journal of Mechanics and Materials in Design, vol. 12, no. 3, pp. 375-386, 2016.

[22] H. Song, K. Yu, X. Li, and J. Han, "Error estimation of load identification based on linear sensitivity analysis and interval technique," Structural and Multidisciplinary Optimization, vol. 55, no. 2, pp. 423-436, 2017.

[23] X. Han, C. Jiang, L. Liu, J. Liu, and X. Y. Long, "Responsesurface-based structural reliability analysis with random and interval mixed uncertainties," Science China Technological Sciences, vol. 57, no. 7, pp. 1322-1334, 2014.

[24] J. R. Klein and S. S. Burkhart, "Identification of essential anatomic landmarks in performing arthroscopic single- and double-interval slides," Arthroscopy: The Journal of Arthroscopic \& Related Surgery, vol. 20, no. 7, pp. 765-770, 2004.

[25] K. Runtemund, G. Cottone, and G. Müller, "Treatment of arbitrarily autocorrelated load functions in the scope of parameter identification," Computers \& Structures, vol. 126, no. 1, pp. 29-40, 2013.

[26] I. Elishakoff, S. Gabriele, and Y. Wang, "Generalized Galileo Galilei problem in interval setting for functionally related loads," Archive of Applied Mechanics, vol. 86, no. 7, pp. 1203-1217, 2016.

[27] C. S. Liu, C. P. Ren, and F. Han, "Study on time-frequency spectrum characteristic of dynamic cutting load based on wavelet regularization," Applied Mechanics and Materials, vol. 577, pp. 196-200, 2014. 
[28] D. W. Gong and Y. Zhang, "Particle swarm optimisation for multi-project location problems with interval profits," International Journal of Modelling, Identification and Control, vol. 8, no. 4, p. 335, 2009.

[29] C. P. Ren and C. S. Liu, "Experimental research on properties of analog materials of coal and rock," Journal of Heilongjiang University of Science and Technology, vol. 24, no. 6, pp. 581584, 2014.

[30] C. Wang, Z. Qiu, and X. Chen, "Uncertainty analysis for heat convection-diffusion problem with large uncertain-butbounded parameters," Acta Mechanica, vol. 226, no. 11, pp. 3831-3844, 2015.

[31] Q. Liu, Y. Li, L. Cao, F. Lei, and Q. Wang, "Structural design and global sensitivity analysis of the composite B-Pillar with ply drop-off," Structural and Multidisciplinary Optimization, vol. 57, no. 3, pp. 965-975, 2018.

[32] Q. Liu, J. Liu, F. Guan, X. Han, L. Cao, and K. Shan, "Identification of the visco-hyperelastic properties of brain white matter based on the combination of inverse method and experiment," Medical \& Biological Engineering \& Computing, vol. 57, no. 5, pp. 1109-1120, 2019. 


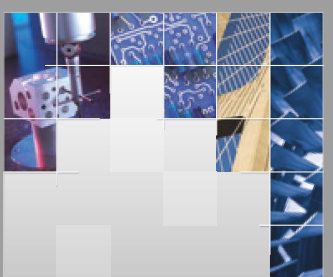

\section{Enfincering}
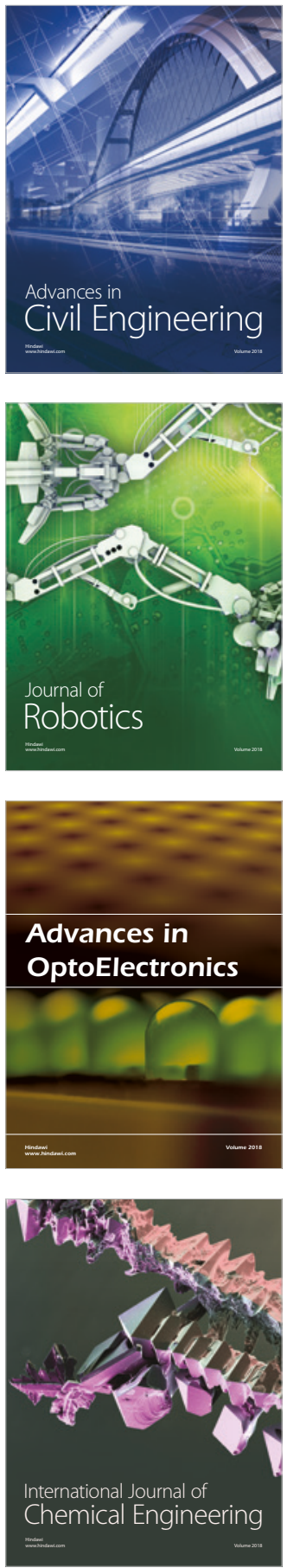

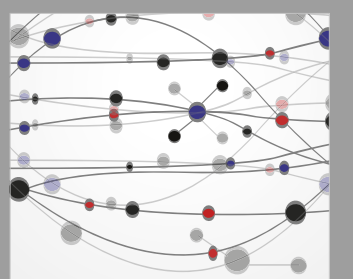

\section{Rotating \\ Machinery}

The Scientific World Journal

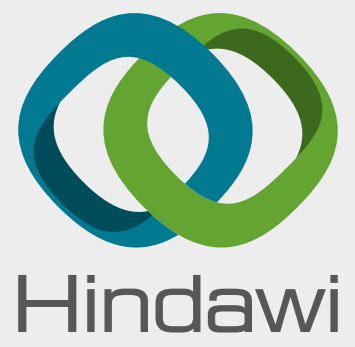

Submit your manuscripts at

www.hindawi.com
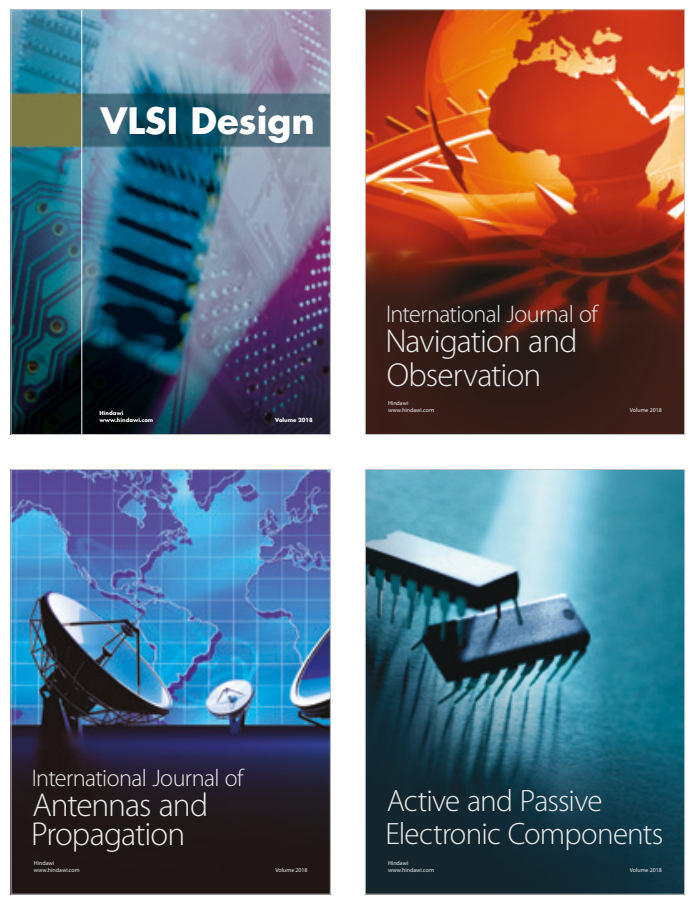
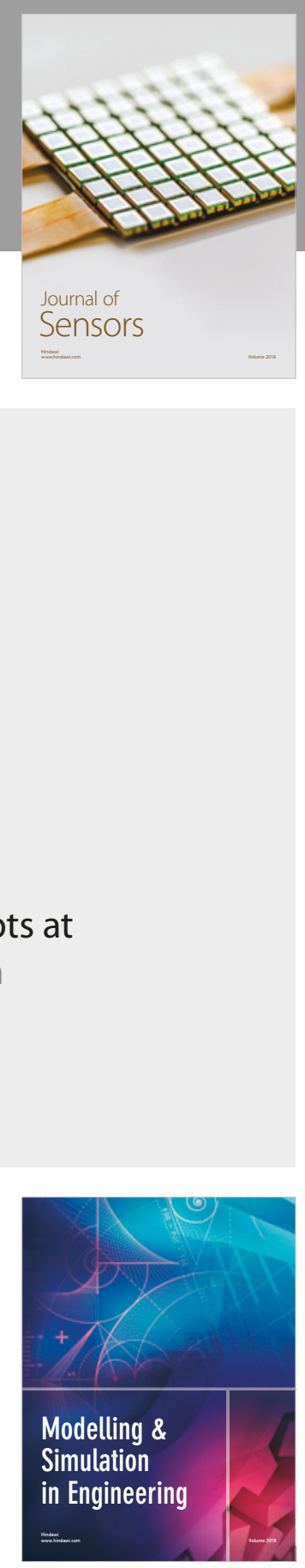

\section{Advances \\ Multimedia}
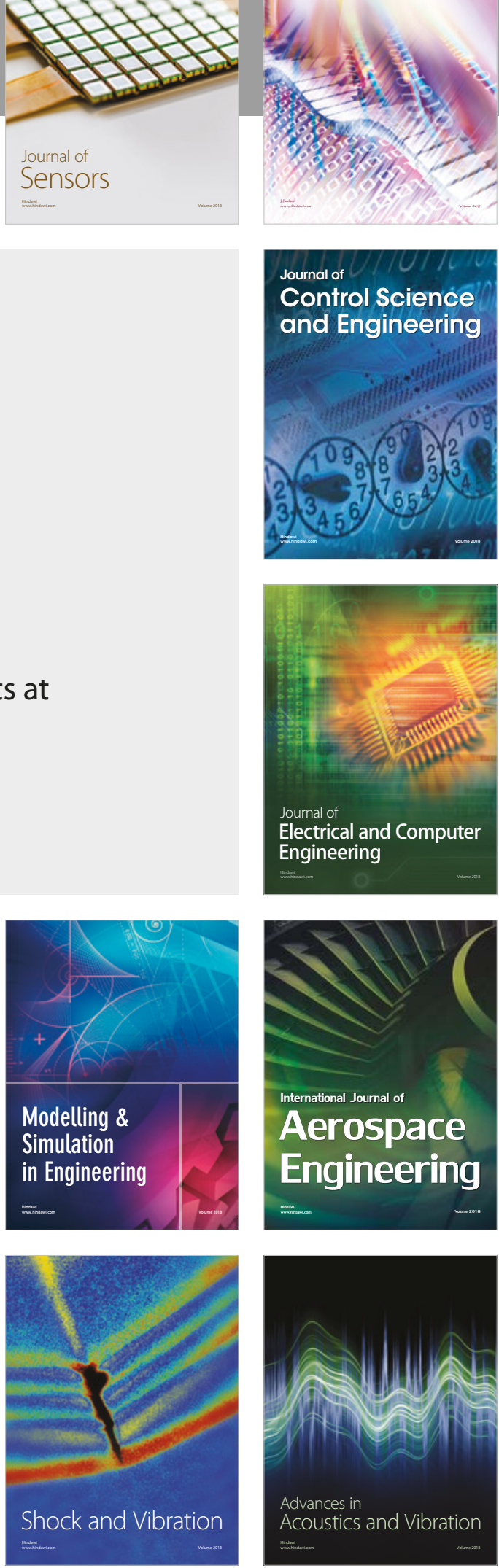